\title{
EXTRAÇÃO DE MATÉRIA ORGÂNICA AQUÁTICA POR ABAIXAMENTO DE TEMPERATURA: UMA METODOLOGIA ALTERNATIVA PARA MANTER A IDENTIDADE DA AMOSTRA
}

\author{
Rosana N. H. Martins de Almeida e Antonio Aparecido Mozeto \\ Departamento de Química, Universidade Federal de São Carlos, CP 676, 13565-905 São Carlos - SP \\ Luiz Fabrício Zara \\ Curso de Química, Universidade Católica de Brasília, Q.S. 07, Lote 01, 72030-170 Brasília - DF \\ André Henrique Rosa e Julio Cesar Rocha* \\ Departamento de Química Analítica, Instituto de Química de Araraquara, Universidade Estadual Paulista "Júlio de Mesquita \\ Filho", CP 355, 14801-970 Araraquara - SP \\ Luciane Pimenta Cruz Romão \\ Universidade Federal do Sergipe, Jardim Rosa Elze s/n, 49100-000 São Cristóvão - SE \\ Ézio Sargentini Junior \\ Instituto Nacional de Pesquisas da Amazônia, CP 478, 69083-000 Manaus - AM \\ Recebido em 1/7/02; aceito em 28/8/02
}

\begin{abstract}
EXTRACTION OF AQUATIC ORGANIC MATTER BY TEMPERATURE DECREASING: AN ALTERNATIVE METHODOLOGY TO KEEP THE ORIGINAL SAMPLE CHARACTERISTICS. In this work was developed an alternative methodology to separation of aquatic organic matter (AOM) present in natural river waters. The process is based in temperature decreasing of the aqueous sample under controlled conditions that provoke the freezing of the sample and separation of the dark extract, not frozen and rich in organic matter. The results showed that speed of temperature decreasing exerts strongly influence in relative recovery of organic carbon, enrichment and time separation of the organic matter present in water samples. Elemental composition, infrared spectra and thermal analysis results showed that the alternative methodology is less aggressive possible in the attempt of maintaining the integrity of the sample.
\end{abstract}

Keywords: aquatic organic matter; humic substances; extraction.

\section{INTRODUÇÃO}

Devido à sua baixa concentração em águas naturais, a extração/ separação da matéria orgânica aquática (MOA) é a primeira e fundamental etapa dos estudos associados à caracterização de suas propriedades. Os principais métodos de separação utilizados levam em consideração três propriedades principais: tamanho, carga e hidrofobicidade. Entretanto, nenhum desses métodos individuais consegue separar todos os principais grupos da MOA, além de oferecerem riscos de contaminação e alteração estrutural dos compostos ${ }^{1,2}$. Desta forma, é importante o desenvolvimento de metodologias alternativas para extração/concentração da MOA, as quais tornem os extratos representativos e mantenham a identidade original da amostra.

O objetivo deste trabalho foi desenvolver uma metodologia para extração da matéria orgânica aquática, utilizando-se apenas o abaixamento de sua temperatura minimizando, assim, a ocorrência de alterações nas características originais da amostra. Para tal, adaptouse um temporizador para controle do abaixamento da temperatura da amostra contida em uma cuba de bebedouro de água comercial. No interior da cuba foi mantida atmosfera inerte (argônio) sob agitação constante. A separação da MOA foi monitorada por medidas dos teores de carbono orgânico total (COT) em diferentes velocidades de abaixamento de temperatura $\left(5-1,6 \mathrm{~mL} \mathrm{~min}^{-1}\right)$ em amostras de água com diferentes concentrações de carbono orgânico (33,0 e $3,0 \mathrm{mg} \mathrm{L}^{-1}$ ). Determinaram-se a porcentagem de recuperação de carbono e o tempo requerido no processo para a separação da MOA. As

*e-mail: jrocha@iq.unesp.br características estruturais da MOA, extraída pela metodologia de abaixamento de temperatura proposta, foi comparada às características das substâncias húmicas aquáticas (SHA) extraídas pela metodologia tradicionalmente utilizada, empregando resina Amberlite XAD 8.

\section{PARTE EXPERIMENTAL}

\section{Reagentes e soluções}

Todos os reagentes utilizados foram de grau e pureza analítica e as soluções preparadas com água desionizada (sistema Milli-Q, Millipore). As soluções ácidas e alcalinas necessárias para a extração das SHA foram preparadas com $\mathrm{HCl}$ 30\% (Merck AG, previamente purificado por destilação) e $\mathrm{NaOH}$ (Merck AG). A resina adsorvente XAD 8 (Serva Feinbiochemica), necessária para a extração das SHA, foi previamente purificada por bateladas sucessivas com soluções de $\mathrm{HCl} 0,5 \mathrm{~mol} \mathrm{~L}^{-1}$, NaOH 0,5 mol L-1 e metanol (duração $24 \mathrm{~h}$ cada batelada).

\section{Coleta e preparo da amostra}

Coletaram-se amostras de água em um afluente do Rio Itapanhaú, pertencente ao Parque Estadual da Serra do Mar, localizado no $11^{\circ}$ Grupo de Unidades de Gerenciamento de Recursos Hídricos (UGRHIs), sétima UGRHI-Baixada Santista, município de BertiogaSP. Parte da amostra foi acidificada até pH 2,0 com solução de $\mathrm{HCl}$ $6,0 \mathrm{~mol} \mathrm{~L}^{-1}$ para posterior extração das SHA com resina XAD 8. No restante da amostra não se adicionaram reagentes, sendo esta mantida sob refrigeração a cerca de $4{ }^{\circ} \mathrm{C}$, até a realização dos experimentos 
para o desenvolvimento da metodologia de extração por abaixamento de temperatura (cerca de 10 dias).

\section{Sistema para extração da matéria orgânica aquática por abaixamento da temperatura}

O aparato para extrair a MOA das amostras de água consistiu de um bebedouro d'água comercial com sistema de refrigeração acoplado a um temporizador "timer", para controlar a velocidade de abaixamento da temperatura. À cuba desse bebedouro, previamente fechada com filme de policloreto de vinila (PVC), foi adaptado um tubo de tygon para manter o sistema sob atmosfera de argônio. Por um orifício no filme de PVC foi introduzido um agitador para homogeneização da amostra. Tanto a cuba do bebedouro, quanto a haste do agitador mecânico foram revestidos com parafilme para evitar contato das superfícies metálicas com a amostra. Este aparato é esquematizado na Figura 1.

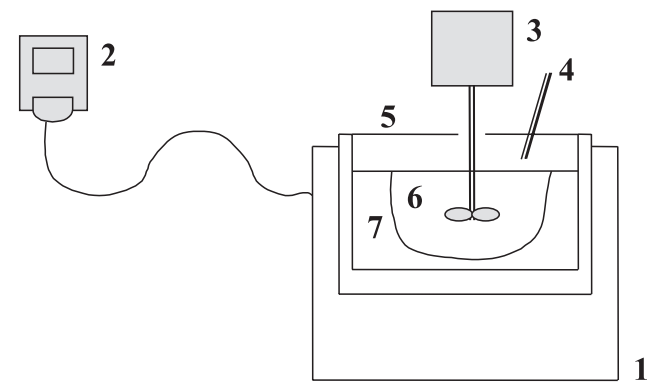

$\begin{array}{ll}\mathbf{1} \text { - Bebedouro d'água comercial (com refrigeração) } & \mathbf{5} \text { - Filme plástico (PVC) } \\ \mathbf{2} \text { - Temporizador } & \mathbf{6} \text { - Extrato orgânico } \\ \mathbf{3} \text { - Agitador mecânico } & \mathbf{7} \text { - Gelo } \\ \mathbf{4} \text { - Entrada de argônio } & \end{array}$

Figura 1. Esquema do sistema proposto para extração da matéria orgânica aquática por abaixamento da temperatura

\section{Procedimento para extração da matéria orgânica aquática por abaixamento da temperatura}

Um volume inicial de $2500 \mathrm{~mL}$ de amostra de água in natura a temperatura de aproximadamente $10{ }^{\circ} \mathrm{C}$ foi transferido para a cuba do bebedouro. O sistema foi fechado com filme de PVC para evitar interferência de $\mathrm{CO}_{2}$ atmosférico, o agitador e o fluxo de gás foram ligados e o congelamento iniciado.

Para determinar a melhor velocidade de congelamento para extração da MOA, o experimento foi feito em quatro diferentes tempos programados como mostra a Tabela 1, utilizando-se duas amostras de água com diferentes concentrações de carbono orgânico (33,0 e 3,0 $\left.\mathrm{mg} \mathrm{L}^{-1}\right)$.

Em intervalos de uma hora foram retiradas alíquotas de 5,0 $\mathrm{mL}$ para determinações dos teores de COT. Após 7 h desligou-se o sistema, o extrato foi retirado, seu volume medido e armazenado sob refrigeração

Tabela 1. Programação do temporizador para os experimentos sobre a influência da velocidade de abaixamento da temperatura na separação da matéria orgânica aquática

\begin{tabular}{lcccc}
\hline Temporizador & \multicolumn{4}{c}{ Experimentos } \\
\cline { 2 - 5 } & $\mathrm{A}$ & $\mathrm{B}$ & $\mathrm{C}$ & $\mathrm{D}$ \\
\hline Ligado (min) & 40 & 20 & 10 & 05 \\
Desligado (min) & 10 & 10 & 10 & 10 \\
\hline
\end{tabular}

para as caracterizações por espectroscopia na região do infravermelho, análise térmica e determinação da composição elementar.

\section{Extração das substâncias húmicas aquáticas por XAD 8, purificação e liofilização}

Após filtração das amostras de água através de papel de filtro qualitativo, fez-se a extração das substâncias húmicas aquáticas utilizando-se procedimento recomendado pela International Humic Substances Society (IHSS) ${ }^{3}$. As SHA foram purificadas por diálise para remoção do excesso de sais dissolvidos, segundo procedimento descrito por Rosa et al. ${ }^{4}$, e liofilizadas.

\section{Caracterizações}

\section{Liofilização}

Segundo Buffle et $a .^{5}$, o procedimento de liofilização não modifica as propriedades da matéria orgânica como absorção a $285 \mathrm{~nm}$, propriedades complexantes, TOC, resíduo seco e massa molecular média. Desta forma, liofilizou-se alíquota de amostra de água em equipamento Savant modelo E-C, para comparar as características da MOA original e da MOA extraída pelos dois procedimentos (abaixamento de temperatura e extração por resina XAD 8).

\section{Determinação do teor de carbono}

As concentrações de carbono foram determinadas pelo método de combustão catalítica sob atmosfera de oxigênio, utilizando analisador de carbono Shimadzu, modelo TOC-5000A.

\section{Espectroscopia na região do infravermelho}

Os espectros de IV foram obtidos de pastilhas preparadas com mistura de iodeto de césio seco a $120{ }^{\circ} \mathrm{C}$ e amostras liofilizadas. As pastilhas foram obtidas submetendo-se essa mistura à uma pressão de $10 \mathrm{t} \mathrm{cm}^{-2}$ em pastilhador de $14 \mathrm{~mm}$ de diâmetro. As medidas foram feitas utilizando-se espectrômetro Nicolet, modelo Impact 400 (região de 400 a $4000 \mathrm{~cm}^{-1}$ ).

\section{Análise térmica}

Segundo procedimento descrito por Leinweber e Shulten ${ }^{6}$, as curvas TG e DTA foram obtidas a partir de cerca de 6,0 mg das amostras liofilizadas, compactadas em cadinho de alumina e calcinadas no SDT 2960 da TA Instruments, sob atmosfera de ar sintético $\left(100 \mathrm{~mL} \mathrm{~min}^{-1}\right)$ e razão de aquecimento de $5{ }^{\circ} \mathrm{C} \min ^{-1}$.

\section{Análise elementar}

Os teores de carbono, hidrogênio, nitrogênio e enxofre das amostras foram determinados pela técnica Dynamic Flash Combustion, em analizador elementar CHNS-O, marca CE Instruments, modelo EA 1110. Os teores de oxigênio foram estimados por diferença entre a soma dos outros elementos e o teor de $\operatorname{cinzas}^{4,7}$.

\section{RESULTADOS E DISCUSSÃO}

\section{Otimização do sistema de extração da matéria orgânica aquática}

Durante a otimização do sistema proposto, as necessidades de alguns procedimentos tornaram-se evidentes, como a utilização de 
agitação constante especialmente para maiores velocidades de congelamento. A diferença de temperatura entre a amostra líquida e a cuba do bebedouro origina um gradiente de temperatura, o qual é responsável pela migração do soluto por difusão térmica. O fluxo convectivo para cima, próximo à interface mais quente e para baixo, próximo à interface mais fria, resulta da diferença de densidade produzida pelo gradiente de temperatura ${ }^{8}$. Como a migração do soluto é dificultada pelo tamanho de suas moléculas, foi necessário auxílio de agitação mecânica constante para favorecer esta migração evitando aprisionamento de soluto na estrutura do gelo formado.

Houve necessidade de trabalhar em sistema fechado com atmosfera inerte, pois se observou elevação nos teores de carbono do extrato quando se utilizou agitação mecânica. Segundo Baker ${ }^{9}$, isso é decorrência da absorção de carbono na forma de $\mathrm{CO}_{2}$ atmosférico dissolvido pela amostra.

\section{Influência da velocidade de abaixamento da temperatura sobre a recuperação relativa de carbono}

Os dados da Figura 2 mostram um aumento relativo nos teores de carbono orgânico ao longo do tempo de processamento da amostra com concentração de carbono orgânico de $33,0 \mathrm{mg} \mathrm{L}^{-1}$, para os quatro experimentos.

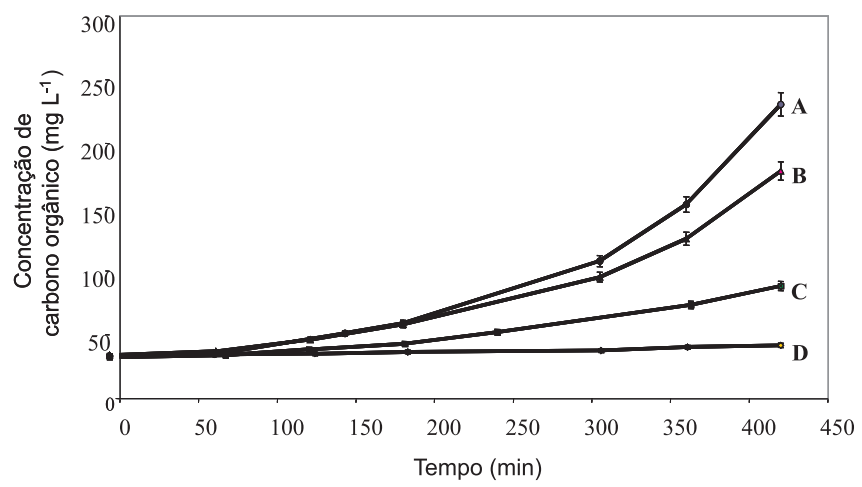

Figura 2. Variação dos teores de carbono orgânico após 7 h de funcionamento do sistema em diferentes velocidades de congelamento (Condições: concentração de carbono orgânico da amostra: 33,0 $\mathrm{mg} \mathrm{mL}^{-1}$; velocidades de congelamento A:5,1; B:4,8; C:3,6; D:1,6 mL $\mathrm{min}^{-1}$ )

Cada um dos quatro experimentos teve como principal diferença a velocidade média de abaixamento de temperatura, sendo o experimento A o mais rápido e o D o mais lento. Observa-se similaridade entre o comportamento do processo nas quatro diferentes velocidades médias de congelamento.

A Figura 2 mostra também, diferença nos valores das concentrações de carbono orgânico, sendo as mais elevadas determinadas no final do experimento A e as menores no final do experimento D. Para estabelecer a melhor velocidade de abaixamento de temperatura, foram calculadas as velocidades médias de congelamento e a porcentagem de recuperação relativa de carbono para os quatro experimentos. Para a amostra com 33,0 $\mathrm{mg} \mathrm{L}^{-1}$ de carbono orgânico, a Figura 3 mostra que a porcentagem de recuperação relativa de carbono entre as velocidades $1,6 \mathrm{~mL} \mathrm{~min}^{-1}$ (experimento $\mathrm{D}$ ), 3,6 $\mathrm{mL}$

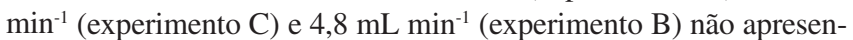
tam grandes diferenças, permanecendo cerca de $98 \%$.

Entretanto, verifica-se um rápido decréscimo na porcentagem de recuperação relativa de carbono com pequeno aumento da velocidade média de congelamento (experimento $\mathrm{A}-5,1 \mathrm{~mL} \mathrm{~min}^{-1}$ ). Isto é caracterizado inclusive pelo visual escurecimento do gelo indicando

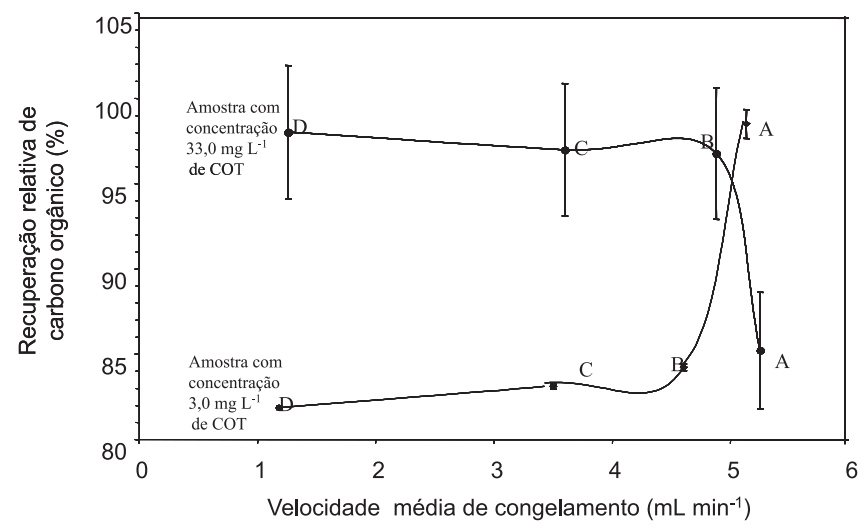

Figura 3. Influência da velocidade média de congelamento sobre a recuperação relativa de carbono orgânico durante o processo de extração de matéria orgânica aquática por abaixamento da temperatura (Condições: concentrações de carbono orgânico nas amostras: 33,0 e 3,0 $\mathrm{mg} \mathrm{L}^{-1}$;

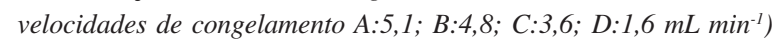

aprisionamento de soluto orgânico. A alta velocidade de congelamento causa formação de gelo mais rápida que a migração das moléculas de soluto para o extrato, aprisionando-as.

Para a amostra com concentração de carbono cerca de 10 vezes menor (3,0 mg L $\left.\mathrm{m}^{-1}\right)$, a Figura 3 mostra que para as velocidades referentes aos experimentos $\mathrm{D}, \mathrm{C}$ e $\mathrm{B}$ não há grandes variações nas porcentagens de recuperação relativa de carbono, as quais são relativamente baixas. Entretanto, a uma velocidade um pouco mais alta (experimento A - 5,1 $\mathrm{mL} \mathrm{min}^{-1}$ ), é possível observar uma brusca elevação nesse valor. Isto indica que para baixas concentrações de carbono orgânico é necessário utilizar velocidades médias de congelamento mais altas pois, a migração do soluto para o centro da cuba é facilitada evitando aprisionamento deste no gelo formado junto à interface cuba/amostra.

Comparação das características da matéria orgânica aquática in natura e dos extratos obtidos pelos dois métodos utilizados

\section{Espectroscopia na região do infravermelho}

À primeira vista, os espectros na região do infravermelho são relativamente simples com poucas bandas largas. Essa simplicidade é mais aparente que real, pois a largura dessas bandas é resultado da superposição das bandas de absorção dos grupos funcionais da matéria orgânica.

A Figura 4 mostra os espectros de absorção na região do infravermelho para os extratos de matéria orgânica obtidos por abaixamento de temperatura, extração em resina XAD 8 e para a amostra in natura (liofilizada).

Em todos os espectros, observa-se uma banda larga na região de $3400 \mathrm{~cm}^{-1}$ devido ao estiramento $\mathrm{OH}$ provavelmente em ligação por ponte de hidrogênio e/ou de $\mathrm{OH}$ de álcoois e/ou fenóis e/ou ácidos carboxílicos. Duas bandas em torno de $3000 \mathrm{~cm}^{-1}$ são observadas levemente superpostas pela banda do estiramento $\mathrm{OH}$. As bandas acima de $3000 \mathrm{~cm}^{-1}$ são referentes ao estiramento $\mathrm{CH}$ de aromáticos e as abaixo de $3000 \mathrm{~cm}^{-1}$, ao estiramento $\mathrm{CH}$ de alifáticos. Nos três espectros observa-se uma pequena banda na região entre 2300 e 2400 $\mathrm{cm}^{-1}$. Essa não é uma banda característica do material estudado, indicando a presença de $\mathrm{CO}_{2}$ como ruído de fundo do equipamento utilizado. Na região de $1700 \mathrm{~cm}^{-1}$, observam-se as bandas características de estiramento $\mathrm{C}=\mathrm{O}$ de cetonas e/ou ácidos carboxílicos. Em torno de $1650 \mathrm{~cm}^{-1}$ a banda observada pode ser atribuída ao 


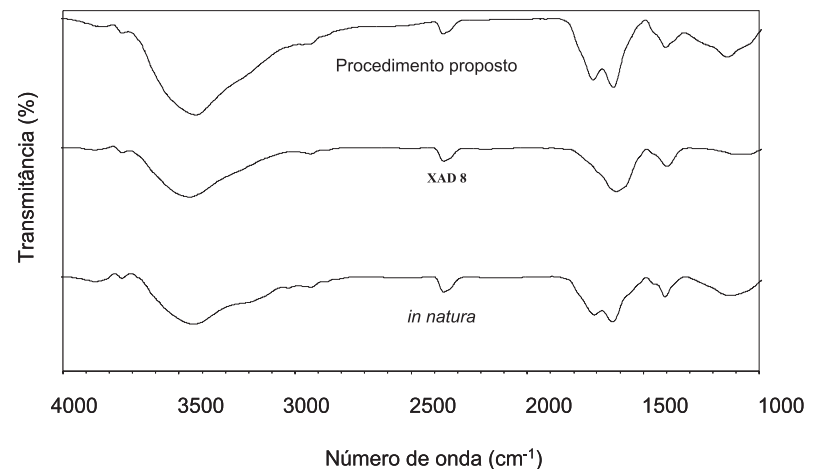

Figura 4. Espectros de absorção na região do infravermelho para os extratos de matéria orgânica obtidos com extração por abaixamento de temperatura, com resina $X A D 8$ e para a amostra in natura

estiramento $\mathrm{C}=\mathrm{C}$ de alquenos e/ou aromáticos. No espectro do extrato orgânico proveniente da resina XAD 8 não é possível distinguir essas duas últimas bandas entre si, sendo observada apenas uma banda mais larga na região de $1650 \mathrm{~cm}^{-1}$. Isso não quer dizer que a banda da carbonila não exista, pois ela pode apenas estar superposta pela banda do estiramento $\mathrm{C}=\mathrm{C}$. Essas observações sugerem um maior grau de similaridade entre o extrato proveniente do congelamento e a amostra in natura. A presença do grupo carboxilato $\mathrm{COO}^{-}$, pode ser notada na banda em torno de $1400 \mathrm{~cm}^{-1}$. Aproximadamente em 1100 $\mathrm{cm}^{-1}$, observa-se uma banda referente ao estiramento da ligação $\mathrm{CO}$ de álcoois e/ou fenóis. O fato dessa banda ser consideravelmente menor para o extrato XAD 8 confirma a idéia de maior similaridade entre as outras duas amostras analisadas.

Com os dados dos espectros de absorção na região do infravermelho foi feita uma análise de correlação linear, isto é, como se correlacionam linearmente as medidas de cada banda em cada número de onda. $\mathrm{O}$ valor da correlação entre os espectros da amostra in natura e do extrato XAD 8 foi 0,93 o que é significativo ao nível de $99,9 \%$. O valor dessa correlação entre os espectros da amostra in natura e do extrato proveniente do processo de congelamento foi 0,95 o que é também significativo ao nível de 99,9\%. Estes valores mostram que o extrato obtido pelo método de abaixamento da temperatura é mais similar com a amostra in natura que o extrato obtido com resina XAD 8.

Ainda com os dados dos três espectros na região do infravermelho, foi feito um tratamento estatístico chamado de análise hierárquica de Cluster ou análise hierárquica de agrupamento. Análise de Cluster é o nome de um grupo de técnicas multivariadas nas quais a proposta primária é agrupar objetos com base em suas características. Este tipo de análise classifica objetos (por exemplo grupos, produtos ou outras entidades) de modo que cada objeto seja muito similar a outros no agrupamento com respeito a alguns critérios de seleção predeterminados. O agrupamento resultante dos objetos deve então exibir alta homogeneidade interna (intra-agrupamentos) e alta heterogeneidade externa (inter-agrupamentos) ${ }^{10}$. Dendograma é o nome dado à representação gráfica dos resultados de um procedimento de Cluster. Esta representação é uma hierarquia (gráfico de árvore) na qual o eixo vertical consiste dos grupos, objetos ou indivíduos e o eixo horizontal consiste do número de agrupamentos formados a cada passo do procedimento. A Figura 5 mostra o dendograma comparativo entre a amostra in natura e os extratos obtidos pelos dois procedimentos aplicados.

A Figura 5 mostra que o extrato orgânico obtido utilizando resina XAD 8 fica logo separado em um grupo diferente, enquanto o extrato orgânico obtido pelo método de congelamento proposto pos- sui uma distância de grupo com relação à amostra in natura quase nula inferindo, assim, o alto grau de similaridade existente entre essas duas amostras.

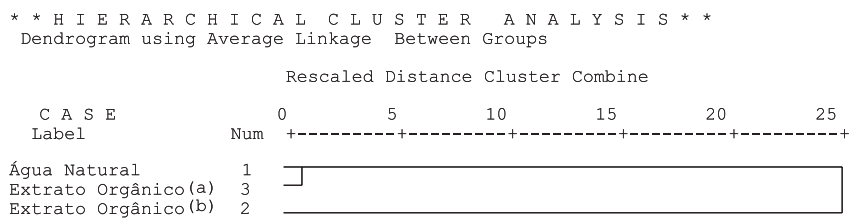

(a) Extrato Orgânico obtido por congelamento

(b) Extrato orgânico obtido por extração com XAD -8

Figura 5. Diagrama do padrão de similaridade entre amostra in natura $e$ os extratos obtidos pelo método de extração por congelamento proposto e por extração com resina $X A D 8$

\section{Análise térmica diferencial e termogravimetria}

Estas duas técnicas foram aplicadas à amostra in natura e aos extratos obtidos pelo método proposto e pelo método de extração com resina XAD 8, também com o objetivo de estabelecer o grau de similaridade dos extratos orgânicos com a água natural. As curvas TG-DTA são mostradas na Figura 6.
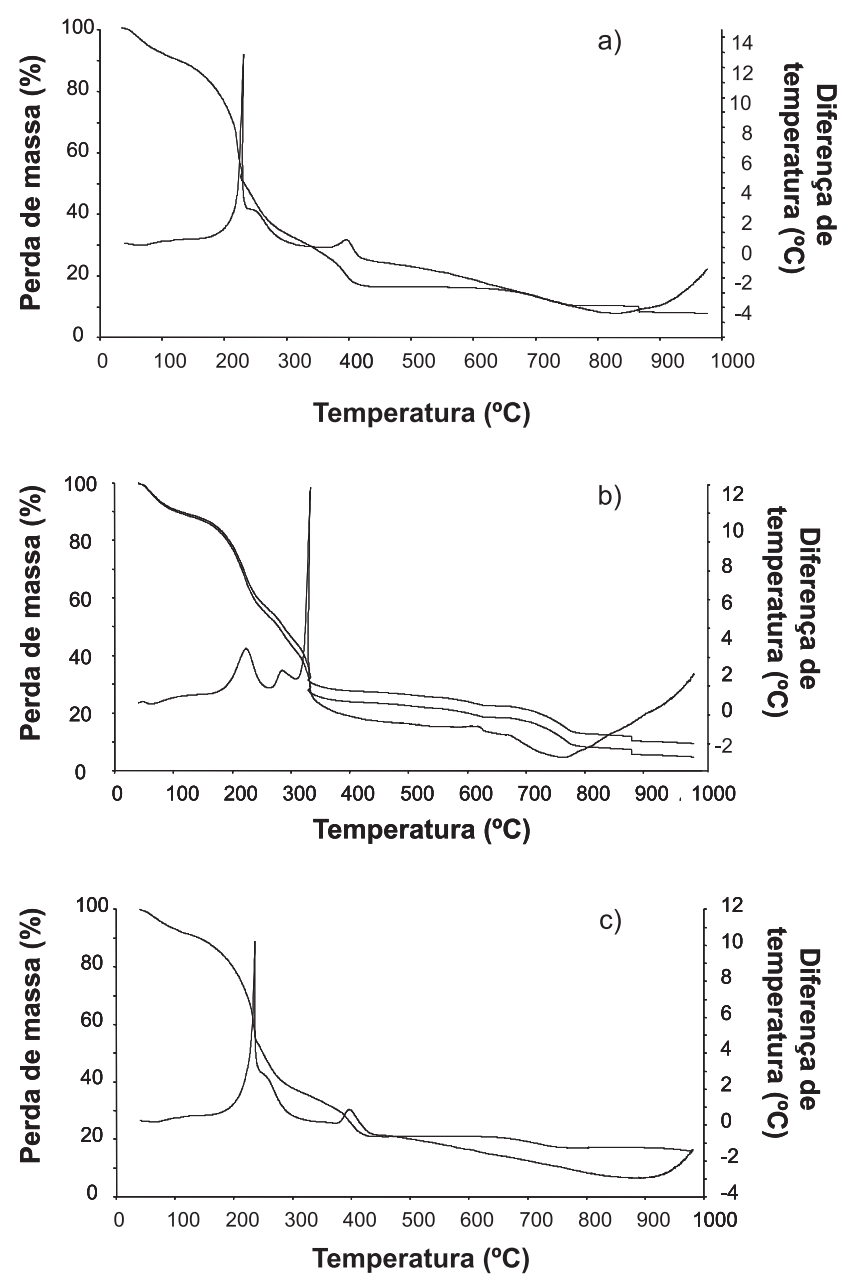

Figura 6. Curvas TG-DTA: a) extrato orgânico obtido pelo método de abaixamento de temperatura (proposto); b) extrato orgânico obtido com resina $X A D 8$ (tradicional); c) amostra in natura 
As curvas TG-DTA simultâneas em atmosfera de ar sintético $\left(100 \mathrm{~mL} \mathrm{~min}{ }^{-1}\right)$ e razão de aquecimento $5{ }^{\circ} \mathrm{C} \mathrm{min}^{-1}$ mostram perda de massa até aproximadamente $150^{\circ} \mathrm{C}$ com pico endotérmico referente à desidratação. Entre 150 e $600{ }^{\circ} \mathrm{C}$ são verificadas etapas de perda de massa com correspondentes picos exotérmicos atribuídos à decomposição térmica oxidativa da parte orgânica com formação de óxidos e/ou carbonatos como resíduo. Entre 290 e $310^{\circ} \mathrm{C}$, segundo Leinweber e Shulten ${ }^{6}$ e Stevenson ${ }^{7}$, ocorre liberação de compostos alifáticos e descarboxilação e, entre 400 e $500{ }^{\circ} \mathrm{C}$ o pico exotérmico é referente à decomposição de aromáticos. Entre 850 e $950{ }^{\circ} \mathrm{C}$ observa-se um pico endotérmico referente à decomposição de carbonatos minerais ${ }^{6}$. Provavelmente, carbonato de cálcio seja decomposto na amostra in natura e no extrato proveniente do processo de congelamento proposto, enquanto no extrato obtido utilizando extração com resina XAD 8 ocorra a decomposição dos carbonatos de cálcio e de sódio (devido à utilização de $\mathrm{NaOH}$ durante o processo de eluição). No intervalo entre 150 e $600{ }^{\circ} \mathrm{C}$ observa-se que o extrato obtido por congelamento apresenta comportamento similar ao da amostra in natura, indicando a não ocorrência de modificações estruturais e de composição na matéria orgânica. O mesmo comportamento não é observado para o extrato obtido via extração com resina XAD 8.

\section{Análise elementar}

Os teores de carbono, hidrogênio, nitrogênio, enxofre e oxigênio e as razões $\mathrm{H} / \mathrm{C}$ e $\mathrm{C} / \mathrm{N}$ para amostra in natura e para os extratos obtidos pelos dois métodos aplicados encontram-se na Tabela 2.

As razões molares $\mathrm{H} / \mathrm{C}$ e $\mathrm{C} / \mathrm{N}$ fornecem indicativos sobre estruturas e grau de humificação da matéria orgânica ${ }^{11}$. O conteúdo de hidrogênio está relacionado com o grau de saturação, isto é, um aumento no teor de hidrogênio indica maior número de carbonos alifáticos $\left(\mathrm{CH}_{2}\right)$ que aromáticos $(\mathrm{C}=\mathrm{C})^{12}$. Mais especificamente, a razão $\mathrm{H} / \mathrm{C}$ é inversamente proporcional à aromaticidade da molécula, e a razão $\mathrm{C} / \mathrm{N}$ é diretamente proporcional à humificação da molécula ${ }^{4}$. Os valores mostrados na Tabela 2 estão de acordo com a literatura $^{4,6}$.

Segundo os dados da Tabela 2, há grande similaridade entre as porcentagens elementares da amostra in natura e do extrato obtido pelo método proposto, enquanto o extrato obtido com a resina XAD 8 possui valores diferentes. Ocorre também uma maior similaridade relacionada à aromaticidade e humificação das moléculas obtidas por congelamento e in natura quando comparadas à molécula do extrato obtido com resina macroporosa XAD 8.

Tabela 2. Composição elementar e razões atômicas H/C e C/N determinadas para os extratos separados por abaixamento da temperatura, utilizando-se resina XAD 8 e amostra in natura

\begin{tabular}{lccccccc}
\hline Amostra & \multicolumn{3}{c}{ Composição elementar (\%)* } & \multicolumn{3}{c}{$\mathrm{H} / \mathrm{C}$} \\
\cline { 2 - 5 } & $\mathrm{C}$ & $\mathrm{H}$ & $\mathrm{N}$ & $\mathrm{S}$ & $\mathrm{O} * *$ \\
\hline Extração por abaixamento da temperatua (proposto) & 43,31 & 1,52 & 5,01 & 0,0 & 50,16 & 0,42 \\
Extração com resina XAD 8 (tradicional) & 48,59 & 1,08 & 6,30 & 0,0 & 44,03 & 0,27 & 10,17 \\
Amostra in natura liofilizada & 42,47 & 1,66 & 5,28 & 0,0 & 50,59 & 0,47 & 9,08 \\
\hline
\end{tabular}

*composição elementar livre de cinzas; **alculado por diferença

\section{CONCLUSÕES}

A metodologia proposta torna possível a separação da matéria orgânica aquática de águas naturais sem adição de reagentes químicos de forma relativamente simples e com baixo custo.

Verificou-se que a agitação constante e a atmosfera inerte são importantes na aplicabilidade do procedimento de extração da matéria orgânica aquática por abaixamento da temperatura.

Também se observou que o processo proposto para separação da matéria orgânica aquática é fortemente influenciado pela velocidade de abaixamento da temperatura e concentração do carbono orgânico na amostra. Para soluções diluídas $\left(3,0 \mathrm{mg} \mathrm{L}^{-1}\right)$ necessitou-se de velocidade de congelamento mais alta $\left(5,1 \mathrm{~mL} \mathrm{~min}^{-1}\right)$ para obter uma porcentagem de recuperação de cerca de $98 \%$. Já para soluções mais concentradas $\left(33,0 \mathrm{mg} \mathrm{L}^{-1}\right)$, a melhor recuperação relativa de carbono orgânico (98\%), foi obtida com menores velocidades médias de congelamento $\left(1,6\right.$ - 4,8 $\left.\mathrm{mL} \mathrm{min}^{-1}\right)$.

Os resultados da composição elementar, espectroscopia na região do infravermelho e análise térmica mostraram que a metodologia proposta minimiza alterações indesejáveis nas características originais da amostra. Esta é a principal contribuição da metodologia proposta, tornando-a interessante quanto aos tratamentos preliminares de matéria orgânica aquática, visando sua caracterização na busca do entendimento de processos ambientais.

\section{AGRADECIMENTOS}

À Fundação de Amparo a Pesquisa do Estado de São Paulo (FAPESP), ao Conselho Nacional de Pesquisa e Desenvolvimento (CNPq) e ao Conselho Nacional de Aperfeiçoamento de Pessoal de Nível Superior (CAPES), por bolsas e suporte financeiro. Ao Prof. Dr. C. Frederico (PUC-Brasília) pelo auxílio no tratamento estatístico dos espectros de infravermelho.

\section{REFERÊNCIAS}

1. Buffle, J.; Complexation Reactions in Aquatic Systems: An Analytical Approach, Ellis Horwood: New York, 1990.

2. Islam, S.; Hemond, H. F.; Anal. Lett. 1991, 24, 1035.

3. Rocha, J. C.; Sargentini Jr., E.; Toscano, I. A. S.; Rosa, A. H.; Burba, P.; J. Braz. Chem. Soc. 1999, 10, 169.

4. Rosa, A. H.; Rocha, J. C.; Furlan, M.; Quim. Nova 2000, 23, 472.

5. Buffle, J.; Greter, F. L.; Haer Di, W.; Anal. Chem. 1977, 49, 216.

6. Leinweber, P.; Shulten, H. R.; Thermochim. Acta 1992, 200, 151.

7. Stevenson, F. J.; Humus Chemistry: Genesis, Composition, Reactions, Wiley: New York, 1994.

8. Jone, A. L. Em Technique of Organic Chemistry: Separation and Purification; Weissberger, A., ed.; Interscience: New York, 1956.

9. Baker, R. A.; Water Res. 1967, 1, 61.

10. Hair, J. F.; Anderson, R. E.; Tatham, R. L.; Black, W. C. Em Multivariate Data Analysis; Prentice-Hall: New Jersey, 1995.

11. Steelink, C. Em Humic Substances in Soil, Sediment and Water. Geochemistry, Isolation and Characterization; Aiken, G. R.; MaCknight, M. D.; Wershaw, R.L.; MaCarthy, P., eds.; Wiley: New York, 1985, p. 457-476.

12. Traina, S. J.; Novak, J.; Smeck, N. E.; J. Environ. Qual. 1990, 19, 151. 